\title{
BMJ Open Quality Improving inpatient warfarin therapy safety using a pharmacist-managed protocol
}

Paul R Daniels, ${ }^{1}$ Dennis M Manning, ${ }^{2}$ James P Moriarty, ${ }^{3}$ Juliane Bingener-Casey, ${ }^{4}$ Narith N Ou, John G O'Meara, ${ }^{5}$ Daniel L Roellinger, ${ }^{3}$ James M Naessens ${ }^{3}$

To cite: Daniels PR, Manning DM, Moriarty JP, et al. Improving inpatient warfarin therapy safety using a pharmacist-managed protocol.BMJ Open Quality 2018;7:e000290. doi:10.1136/ bmjoq-2017-000290

Received 14 December 2017 Revised 27 February 2018 Accepted 24 March 2018
Check for updates

${ }^{1}$ Division of General Internal Medicine, Mayo Clinic, Rochester, Minnesota, USA ${ }^{2}$ Division of Hospital Internal Medicine, Mayo Clinic, Rochester, Minnesota, USA ${ }^{3}$ Department of Health Sciences Research, Mayo Clinic, Rochester, Minnesota, USA ${ }^{4}$ Department of Surgery, Mayo Clinic, Rochester, Minnesota, USA

${ }^{5}$ Department of Pharmacy Services, Mayo Clinic, Rochester, Minnesota, USA

Correspondence to

Dr Paul R Daniels;

daniels.paul@mayo.edu

\section{ABSTRACT}

Introduction Safe management of warfarin in the inpatient setting can be challenging. At the Mayo Clinic hospitals in Rochester, Minnesota, we set out to improve the safety of warfarin management among surgical and non-surgical inpatients.

Methods A multidisciplinary team designed a pharmacistmanaged warfarin protocol (PMWP) which designated warfarin dosing to inpatient pharmacists with guidance from computerised dosing algorithms. Ordering this protocol was ultimately designed as an 'opt out' practice. The primary improvement measure was frequency of international normalised ratio (INR) greater than 5; secondary measures included adoption rate of the protocol, a counterbalance INR metric (INR $<1.7$ three days after first inpatient warfarin dose), and complication rates, including bleeding and thrombosis events. An interrupted time series analysis was conducted to compare outcomes. Results Among over 50000 inpatient warfarin recipients, the PMWP was adopted for the majority of both surgical and non-surgical inpatients during the study period (1 January 2005 to 31 December 2011). The primary improvement measure decreased from $5.6 \%$ to $3.4 \%$ for medical patients and from $5.2 \%$ to $2.4 \%$ for surgical patients during the preimplementation and postimplementation periods, respectively. The INR counterbalance measure did not change. Postoperative bleeding decreased from $13.5 \%$ to $11.1 \%$ among surgical patients, but bleeding was unchanged among medical patients.

Conclusion Our PMWP led to achievement of improved INR control for inpatient warfarin recipients and to less near-term bleeding among higher risk, surgical patients.

\section{INTRODUCTION}

\section{Problem description}

Maintaining stable anticoagulant effect with warfarin can be difficult; challenged by drug interactions, medical comorbidities and diet, warfarin management can result in subtherapeutic or supratherapeutic anticoagulation and corresponding complications. ${ }^{1}$ Despite availability of new oral anticoagulants, warfarin is still widely used and hospital practices will continue to care for patients taking this medication. $^{2-4}$

Change regarding inpatient warfarin management at the Mayo Clinic Rochester hospitals began with observations by the Mayo Clinic Mortality Review Committee of adverse patient events related to warfarin. As part of an Institute for Healthcare Improvement (IHI) mortality review in 2003, 100 inpatient deaths were reviewed, particularly 'Box 4'- the 59 decedents who were admitted to general care (non-intensive care unit (ICU)) units for full therapeutic (non-hospice) care. ${ }^{5}$ Of seven cases revealing some opportunity for systems improvement were two cases in which warfarin-induced supratherapeutic international normalised ratio (INR) $>5$ was a contributing factor.

Each year at the Mayo Clinic in Rochester, Minnesota, warfarin is given to approximately 5000 non-surgical inpatients and over 4500 surgical inpatients at either Saint Mary's or Rochester Methodist Hospital. In a preliminary analysis, $5.5 \%$ of hospitalised patients receiving at least one inpatient dose of warfarin subsequently had an INR $>5$ during the same hospital stay. We chose this metric since an excessively supratherapeutic level of anticoagulation increases the risk of bleeding complications. ${ }^{6}$ In addition, the IHI recommended identifying episodes of INR $>5$ for warfarin recipients by use of a global trigger tool. ${ }^{7}$

\section{Available knowledge}

A nationally representative sample of inpatient stays estimated that $10 \%$ of drug-related adverse outcomes stemmed from anticoagulants. ${ }^{8}$ Beginning in 2008, the Joint Commission made anticoagulant therapy a specific National Patient Safety Goal, stating that hospitals should 'use approved protocols for the initiation and maintenance of anticoagulant therapy. ${ }^{, 9}$ Prior to initiation of our project, published reports outlined new systems approaches to inpatient anticoagulation management. For example, Dager et al reported decreases in supratherapeutic INR among inpatient warfarin recipients when pharmacists provided daily dosing recommendations. ${ }^{10}$ Using administrative databases, Bond and Raehl demonstrated that in hospitals 
in which pharmacists provided management, warfarin-related outcomes were improved. ${ }^{11}$ We determined that our process redesign should involve management input from pharmacists.

\section{Rationale}

The Mayo Clinic inpatient practice encompasses a diverse and large group of prescribers, including trainees, introducing variability in warfarin management. We reasoned that a standardised protocol would improve safety. With this process, similar patients should be more likely to receive similar management; standardisation could decrease variation and expertise (pharmacists) could improve performance. Iterative assessments could refine the protocol and be rapidly implemented.

\section{Specific aims}

The primary aim was to reduce the frequency of excessive supratherapeutic anticoagulation (INR $>5$ ) among inpatient warfarin recipients through a pharmacist-managed warfarin protocol (PMWP). Secondarily, we aimed to reduce bleeding and thrombosis events, while not increasing subtherapeutic anticoagulation as gauged by a counterbalance measure. The purpose of the current report is to share the results of these analyses. This report follows the Standards for Quality Improvement Reporting Excellence format for presenting quality improvement projects. $^{12}$

\section{METHODS \\ Context}

The sites for this project were the Mayo Clinic hospitals in Rochester, Minnesota-Saint Mary's Hospital, Rochester Methodist Hospital, and the inpatient Mayo Clinic Psychiatry and Psychology Treatment Center. These hospitals represent the total inpatient practice at Mayo Clinic Rochester. The Mayo Clinic Department of Medicine Quality Office and the Department of Surgery Quality Committee sponsored the project; the Mayo Clinic Clinical Practice Quality Oversight Subcommittee endorsed spread across departments. The present analysis consists of adult inpatients (age $\geq 18$ ) given at least one inpatient dose of warfarin and discharged between 1 January 2005 and 31 December 2011.

\section{Interventions}

A team of physicians, pharmacists, nurses, laboratory technicians, statisticians, information technology personnel and project managers was created; the Define, Measure, Analyze, Improve and Control (DMAIC) framework was used and the project team met on a scheduled basis. Phases of DMAIC were explicitly identified as the project progressed, from a shared understanding of the definition of the problem, through measurement of baseline performance, to analysis of possible interventions and implementation of the improvement phase. A control plan was determined after full implementation. Project managers organised and facilitated team meetings/tasks.
With many team members engaged in clinical practice, project managers were available to drive project advancement. Accountability for the DMAIC process was to Mayo Clinic quality oversight committees.

\section{Pharmacist-managed warfarin protocol}

During the DMAIC process (Analyze phase), the decision was made to develop a PMWP. Under the PMWP, pharmacists would monitor interacting medications and changes in patient status, and manage warfarin dosing by a protocol. The protocol underwent multiple revisions to arrive at the current version; this was published in 2013. ${ }^{13}$ Given the primary aim of the project, our team prospectively monitored the performance of the protocol, analysing the cases in which patients on the protocol had an INR $>5$ to identify potential reasons for these defects. This information was then used in new iterations of the protocol. For example, analysis showed the presence of certain risk factors among those with INR $>5$ on protocol, including liver disease, poor nutritional states and diarrheal illness. The protocol was modified by prescribing lower initial inpatient warfarin doses to patients with these high sensitivity risk factors.

Prior to the introduction of a PMWP, inpatient warfarin management was by the primary team caring for the patient. Under standard practice at Mayo Clinic hospitals, warfarin orders must be placed daily; standing orders are not permitted. Dose selection and frequency of INR monitoring were by the patient's providers. Under the PMWP the provider specifies a warfarin protocol (ie, new, resume, or continuation protocol), indication(s) and the goal INR range, and preadmission dose. The PMWP is supported by electronic tools that automate important functions related to dosing and monitoring. A computerised warfarin system was developed with capabilities to calculate dose based on an algorithm using clinical factors and INR values, automate lab ordering and monitor for unexpected changes in lab results (eg, if INR increases beyond a specified number, alert pharmacist to repeat test 9-12 hours later). Additionally, the pharmacist uses the warfarin system to write daily progress notes. The pharmacist assesses factors that can influence warfarin dosing including drug interactions, comorbidities (eg, heart failure, diarrhoea) and dietary intake and selects a first inpatient dose. On day 2 and thereafter, a computerised algorithm recommends a dose range based on the INR change in the past 24 hours and the pharmacist selects the dose for the day. If in the pharmacist's clinical judgement a deviation from the suggested dose range is warranted, the primary team is contacted. Daily INR values were measured while on the protocol. ${ }^{13}$ Warfarin dosing and the pharmacists' notes are available to all providers in the electronic medical record.

The General Internal Medicine and Hospital Internal Medicine Divisions (general medicine services) endorsed usage of the protocol on their services, available as an opt-in process, in January 2007. Weekly prospective data were collected on all warfarin patients (on and 
off protocol) and the frequencies of INR $>5$ compared between the two groups and to pre-PMWP performance; reduction in INR $>5$ was significant with the PMWP. During the next year (mid-2007 to late 2008), presentations on the PMWP were made to practice leadership for each inpatient department or division, including the entire department of medicine, surgical departments, neurology, oncology, psychiatry and family medicine. These presentations explained the PMWP process and informed each new area regarding their baseline performance (INR $>5$ ). Once the entire hospital practice had endorsed availability of the protocol, it was made opt-out (July 2009).

\section{Study of the intervention}

We conducted a prospective pilot of the PMWP on the general medicine services with measurement of our primary outcome on a weekly basis to determine if it should be spread to other hospital services. During this phase, patients managed by the PMWP were compared with usual care. After implementation across the inpatient practice, the design of our analysis was a retrospective cohort with patients on warfarin identified through administrative billing systems; inference of effect was assessed through an interrupted time series analysis. Since our design was not a randomised clinical trial, the postimplementation phase includes patients not on the PMWP. To minimise bias, we opted to group all patients on warfarin (both on and off PMWP) for analysis. By comparing preimplementation with postimplementation results, during which there is a 'steady state' usage of the protocol, we infer that that the differences are due to the intervention. Patients on new, resume and continuation dosing aggregated as well.

\section{Measures}

Patient records were linked to the clinical system tracking all medication orders for verification. Clinical databases containing laboratory test values and ordered medications were also linked. Administrative systems were also used for demographic information, secondary diagnoses and other provided services.

The primary outcome measure was defined as an INR $>5$ following administration of at least one dose of warfarin during an inpatient episode of care. All INR measurements from initiation of warfarin based on medication orders until the earlier of 10 days following the last warfarin administration or hospital discharge were considered for detecting elevated INR.

Secondary outcomes included bleeding, thrombotic events and a low INR counterbalance measure. Bleeding and thrombotic events were identified using multiple elements from clinical and administrative databases and were captured if they occurred during warfarin administration or during the subsequent 10 days. Bleeding events for medical patients were identified using the presence of two or more of the following three elements: (A) selected secondary diagnoses indicating haemorrhage,
(B) significant reduction of haemoglobin or haematocrit, or (C) administration of blood products or factor medications. For surgical patients, a fourth element was included for haemorrhage control procedures (such as return to the operating room for bleeding control), with a 'bleed' still defined as the presence of any two criteria. Bleeding diagnoses included cerebral haemorrhage (International Classicification of Diseases Ninth Revision (ICD-9) diagnosis codes 430-432.99), gastrointestinal haemorrhage and 'other' bleeds. Gastrointestinal haemorrhage was identified by diagnosis codes used by the Agency for Healthcare Quality and Research (AHRQ) Patient Safety Indicator (PSI) of death rate among surgical inpatients with serious treatable complications. ${ }^{14}$ Diagnosis codes 285.1, 459.0, 568.81, 958.2 and 998.11 comprised the 'other' category. A significant haemoglobin and haematocrit decline was defined as a drop of haemoglobin of $4 \mathrm{~g} / \mathrm{dL}$ or drop of haematocrit of $12.0 \%$, a level used in multiple cardiac intervention trials. ${ }^{15}$ Potential bleeding events based on blood product use were identified by the occurrence of 2 or more units of red blood cells within a 2-day period or 1 or more units in a 2-day period of packed red blood cells, fresh frozen plasma or cryoprecipitate. Additionally, any dose of factor medication was considered an indication of a potential bleed. Baseline values of haemoglobin and haematocrit were defined as the most recent value prior to warfarin management when available; otherwise, it was the value measured the day warfarin management was initiated in the hospital. If no measurements were found in this manner (about 13\% of observations), baseline values were imputed based on the low end of normal ranges (haemoglobin: 13.5 for men, 12.0 for women; haematocrit: 38.8 for men, 34.9 for women). Haemorrhage control procedures were defined based on the AHRQ PSI for postsurgical bleeding. ${ }^{16} \mathrm{~A}$ manual review of a sample of medical cases indicated that our bleeding algorithm has good sensitivity $(93.9 \%)$ but only fair positive predictive value $(46.2 \%) .{ }^{17}$ Although this performance is not ideal, we feel that we are consistently identifying patients with high likelihood of adverse events.

Thrombotic events included deep vein thrombosis (DVT), pulmonary embolism (PE), cerebral occlusion and myocardial infarction (MI). Events were identified using hospital-acquired secondary diagnosis coding. ${ }^{18-20}$ Event timing was based on the date of key diagnostic tests determined using CPT4 codes or lab values. Hospital-acquired thrombotic events were only included when a corresponding timing element was identified during or after warfarin management. DVT events were defined as ICD-9 codes 451.11, 451.19, 451.2, 451.81, 451.9, 453.40, 453.41, 453.42, 453.8 and 453.9. Timing of DVT events was based on dates of selected diagnostic scans (CPT4 codes 85379, 85378, 93926, 93925 and 93922). Timing of PE events (ICD-9 diagnosis codes: 415.1, 415.11 and 415.19) was determined by CPT4 codes 85379, 85378, 75741, 75743, 75746, 71250,71560 and 71270. Cerebral occlusions were identified using diagnoses 433-434.99 and date of occurrence 
using CPT4 codes 70450, 70460 and 70 470. Diagnosis codes identifying MI were 410.X1. Timing of MIs was determined by an elevated creatine kinase-muscle/brain lab value $(>6.7$ for men and $>3.8$ for women) or troponin value $(>0.03)$. The counterbalance measure of INR was defined as the per cent of patients with an INR $<1.7$ after 3 days of warfarin management among those still on anticoagulation. Other secondary measures included orders for warfarin being held and days with no INR measurements.

\section{Analyses}

All study variables were analysed using an interrupted time series analysis. Control charts of each outcome were created allowing for different limits for each study time period: (A) preimplementation (2005-2006), (B) roll-out (2007-2009) and (C) postimplementation (2010-2011). For the control charts, $99.7 \%$ CIs (3 sigma) were calculated around each mean monthly outcome measure. The extent of implementation was assessed by the percentage of warfarin patients on PMWP. Outcome measures were plotted by month of hospital discharge. Overall effects of the warfarin protocol on high INR, low INR, warfarin being held and missing INR were tested comparing differences in monthly mean values between the preintervention versus postintervention time periods using the Wilcoxon rank-sum test. All other outcome measures were compared by the complete preimplementation and postimplementation intervals using the $\mathrm{X}^{2}$ test or Fisher's exact test, where appropriate. For rare events such as bleeding and thrombosis a $\mathrm{X}^{2}$ test was performed comparing counts in the preperiods versus postperiods rather than monthly values. All analyses were performed separately for surgical and non-surgical patients. A significance level of 0.05 was used. The roll-out period was not included in statistical analyses. All analyses were performed using SAS V.9.2.

\section{Ethical considerations}

The analysis was submitted to the Mayo Clinic Institutional Review Board and judged to be exempt from research review. None of the project team was deemed to have conflicts of interest. By prospectively evaluating the impact of the PMWP in the pilot practice area (general medicine) we could ascertain that an improvement was observed and could advocate for expansion of the process change to other practice areas.

\section{RESULTS}

A total of 24624 inpatient medical encounters and 33009 inpatient surgical encounters with warfarin administration during the hospitalisation were identified from 2005 to 2011. Of these, 335 were of children and another 258 $(0.45 \%)$ could not be verified with warfarin orders in clinical databases. The analysis population consisted of 24352 inpatient medical encounters and 32688 inpatient surgical encounters. There were 15873 inpatient encounters in the preimplementation period, 25202 in the roll-out period and 15965 in the postimplementation period. Characteristics of patients during the three time periods are depicted in table 1 .

Among medical patients, there was a significant increase in the per cent of admissions labelled emergent or urgent in the postimplementation time frame, as well as an increase in discharges to skilled nursing facilities. Privately insured decreased while government insurance increased. A higher proportion of medical warfarin patients were on general medicine, neurology and thoracic services when comparing postimplementation and preimplementation periods. Among the surgical patients the proportion of orthopaedic patients increased from the preimplementation to postimplementation time frame. Patients on average were older, more often government insured and discharged with healthcare assistance. Mean length of stay for surgical patients on warfarin dropped from 10.0 days during preimplementation to 8.6 during postimplementation. Use of the ICU among surgical patients decreased over time.

PMWP use is displayed in figure 1. For medical patients, in the roll-out period a total of $4411(42.8 \%)$ were under the pharmacist protocol at some point during the hospital encounter, increasing to $5703(83.2 \%)$ in the postimplementation period. For surgical patients, 2909 (31.9\%) were managed by the pharmacist protocol at some point during the roll-out, increasing to $6225(68.2 \%)$ in postimplementation.

The monthly percentage of INR $>5$ encounters compared with the total number of encounters is shown in figure 2. A downward shift can be seen in both the roll-out and postperiods moving from 5.6\% among medical patients and $5.2 \%$ among surgical patients during the preimplementation phase to $3.4 \%$ among medical patients and $2.4 \%$ among surgical patients during postimplementation.

The percentage of patients maintaining the counterbalance measure remained stable throughout the study time frame (figure 3). As displayed in table 2, there were no significant changes in bleeding events among medical patients; there was a significant decrease in postoperative bleeding events between the preimplementation and postimplementation.

There were significantly more ischaemic strokes in the postimplementation period among medical patients. Among surgical patients, we also saw improvements in the rate of low INR values and fewer thromboses. The percentage of patients with at least 1 day without INR value substantially decreased during the study time frame among both medical and surgical patients. Surgical patients had substantially fewer 'hold' orders after implementation.

\section{DISCUSSION}

\section{Summary}

Implementation of a PMWP for inpatients at Mayo Clinic Rochester Hospitals resulted in several key successes. The primary aim to reduce the frequency of excessive supratherapeutic anticoagulation was achieved. 
Table 1 Patient and stay characteristics for hospitalised medical patients and surgical patients on warfarin, Mayo Clinic Rochester Hospitals, 2005-2011

\section{A. Medical patients}

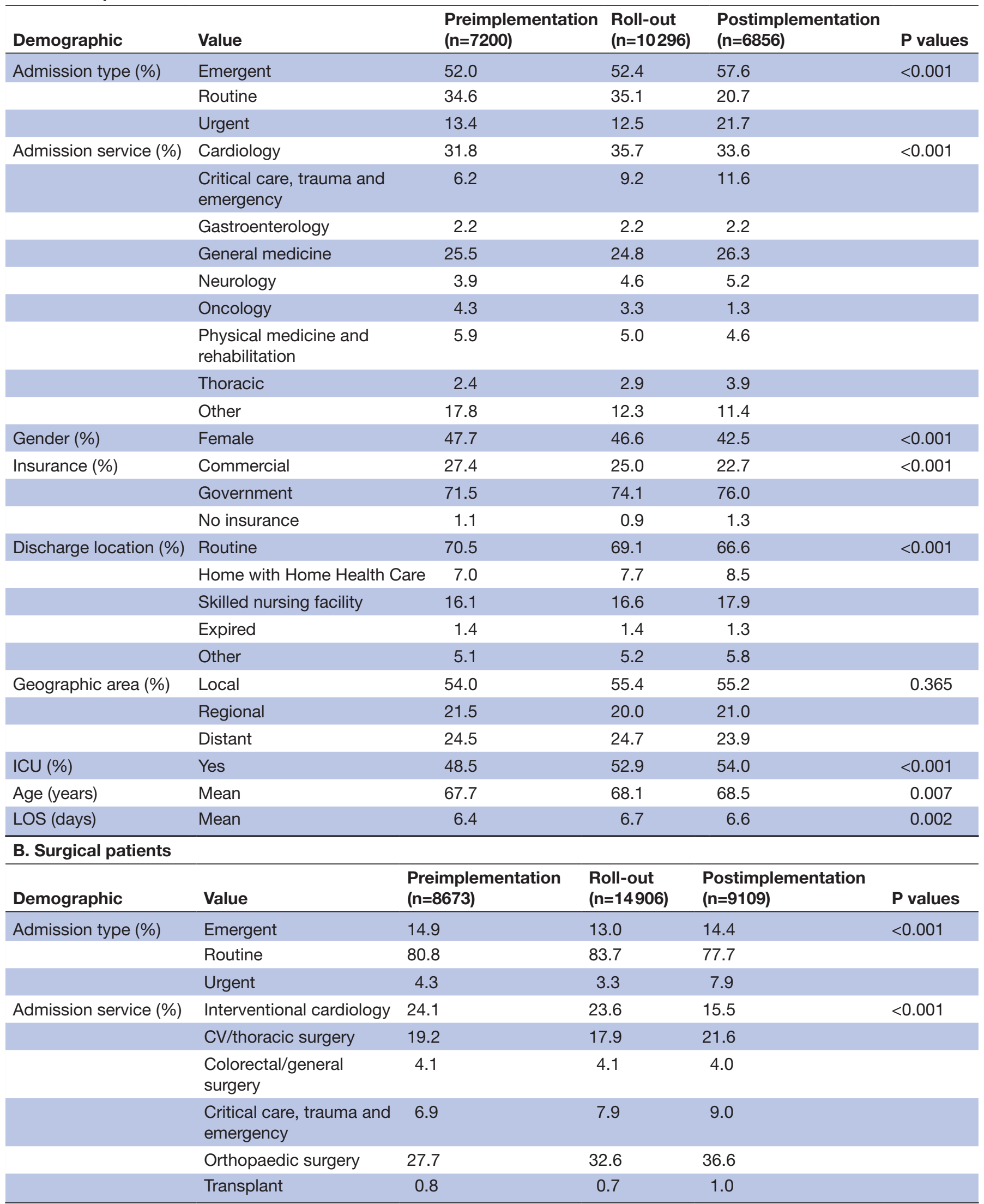


Table 1 Continued

\section{B. Surgical patients}

\begin{tabular}{|c|c|c|c|c|c|}
\hline Demographic & Value & $\begin{array}{l}\text { Preimplementation } \\
(\mathrm{n}=8673)\end{array}$ & $\begin{array}{l}\text { Roll-out } \\
(n=14906)\end{array}$ & $\begin{array}{l}\text { Postimplementation } \\
(\mathrm{n}=9109)\end{array}$ & $P$ values \\
\hline & Vascular surgery & 4.4 & 4.1 & 3.6 & \\
\hline & $\begin{array}{l}\text { Gynaecology/OB and } \\
\text { urology }\end{array}$ & 3.4 & 2.4 & 2.22 & \\
\hline & Other & 9.3 & 6.7 & 6.6 & \\
\hline Gender (\%) & Female & 44.8 & 44.4 & 45.3 & $<0.001$ \\
\hline \multirow[t]{3}{*}{ Insurance (\%) } & Commercial & 36.9 & 36.7 & 35.0 & $<0.001$ \\
\hline & Government & 61.9 & 62.1 & 64.0 & \\
\hline & No insurance & 1.3 & 1.2 & 1.0 & \\
\hline \multirow[t]{5}{*}{ Discharge location (\%) } & Routine & 68.6 & 68.8 & 63.4 & $<0.001$ \\
\hline & $\begin{array}{l}\text { Home with Home } \\
\text { Health Care }\end{array}$ & 4.9 & 6.3 & 8.0 & \\
\hline & Skilled nursing facility & 19.2 & 19.4 & 22.1 & \\
\hline & Expired & 1.0 & 0.8 & 1.0 & \\
\hline & Other & 5.3 & 4.7 & 5.5 & \\
\hline \multirow[t]{3}{*}{ Geographic area (\%) } & Local & 26.2 & 27.1 & 27.7 & 0.365 \\
\hline & Regional & 26.5 & 25.9 & 26.9 & \\
\hline & Distant & 47.4 & 47.0 & 45.4 & \\
\hline ICU (\%) & Yes & 61.7 & 57.6 & 52.9 & $<0.001$ \\
\hline Age (years) & Mean & 64.7 & 64.9 & 65.7 & 0.007 \\
\hline LOS (days) & Mean & 10.0 & 8.9 & 8.6 & 0.002 \\
\hline
\end{tabular}

CV, cardiovascular; ICU, intensive care unit; LOS, length of stay; OB, obstetrician.

Significant decreases in postoperative haemorrhage and thrombosis were also seen among surgical patients. Strengths of the project include extension of the process change to all areas of the Mayo Clinic hospital practice and the large numbers of patients impacted and analysed.

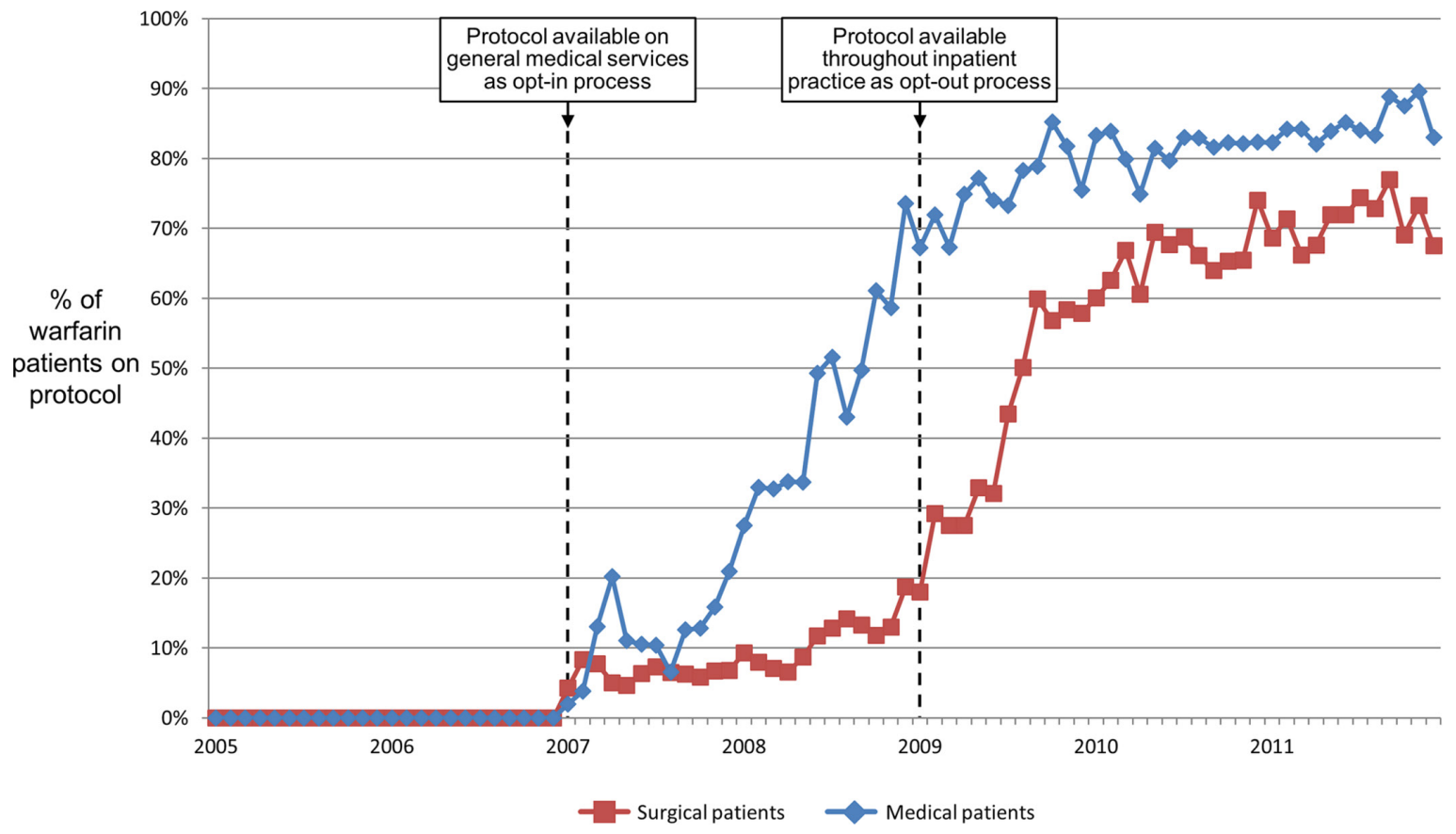

Figure 1 Use of pharmacist-managed warfarin protocol among hospitalised patients. 
A. Medical Patients

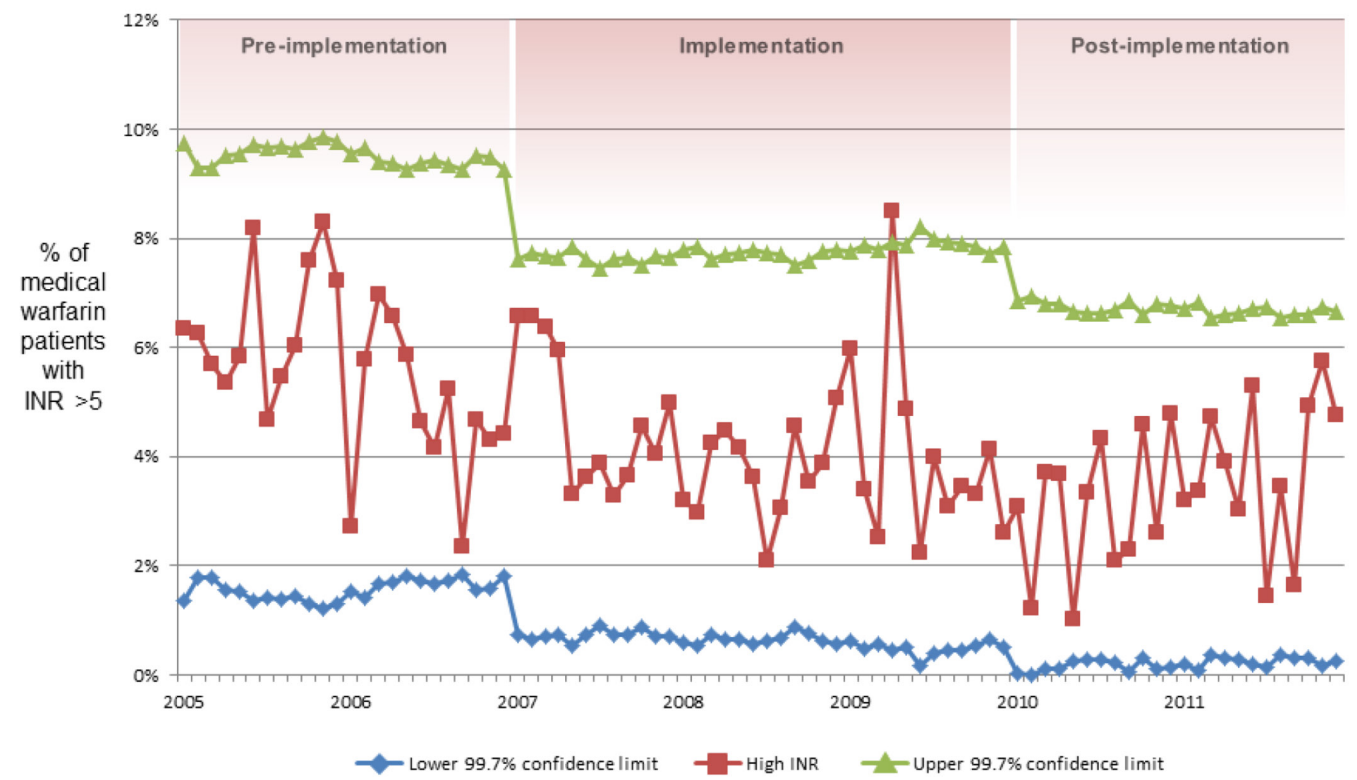

\section{B. Surgical Patients}

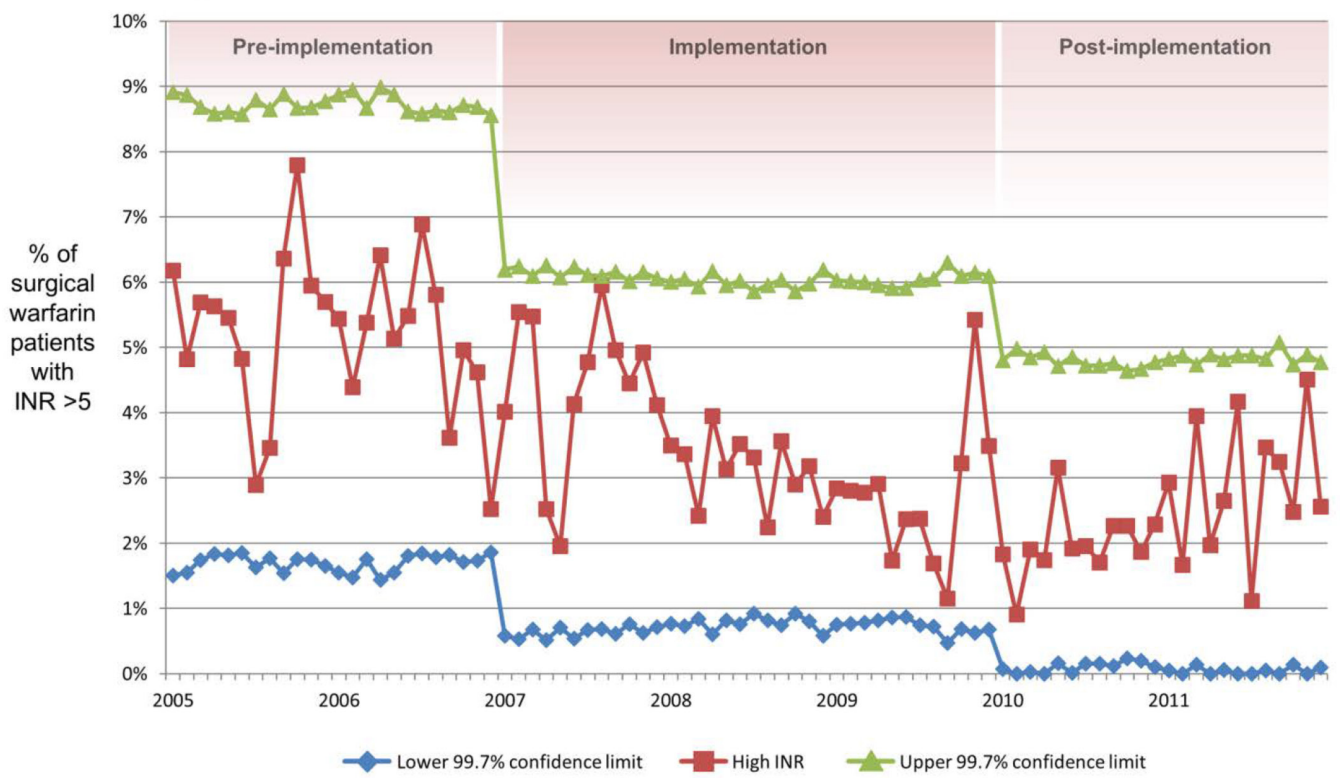

Figure 2 Per cent of inpatient warfarin recipients with international normalised ratio (INR) greater than 5 (high INR) from preimplementation to postimplementation phases.

\section{Interpretation}

This project was not a randomised trial isolating specific process changes regarding warfarin management. That is, whether the primary outcome improvement was due to the impact of a pharmacist or to use of a standard dosing algorithm alone cannot be determined. The design of the project was not to separate those changes but to assess the effect of combined process changes. The analytic model and large number of patient experiences allow reasonable inference that the overall process change improved care. Secular trends may have had some influence on results in the surgical population; for example, while some of the perioperative success may have been achieved by providing timely warfarin orders independent of the surgical workload, other practice changes, such as energy devices and medication given in the operating room may have had an influence on the postoperative haemorrhage and thrombosis results.

Our results are congruent with other published reports of the impact of interventions on inpatient warfarin management by similar metrics. ${ }^{21-24}$ For example, Wong et al reported that involving pharmacists in warfarin initiation dosing significantly reduced the proportion with INR $>4$ (from $27 \%$ of 26 patients managed by usual care to $2 \%$ of 144 patients managed by pharmacists). ${ }^{21}$ Gouin-Thibault et al compared usual care (199 patients) 


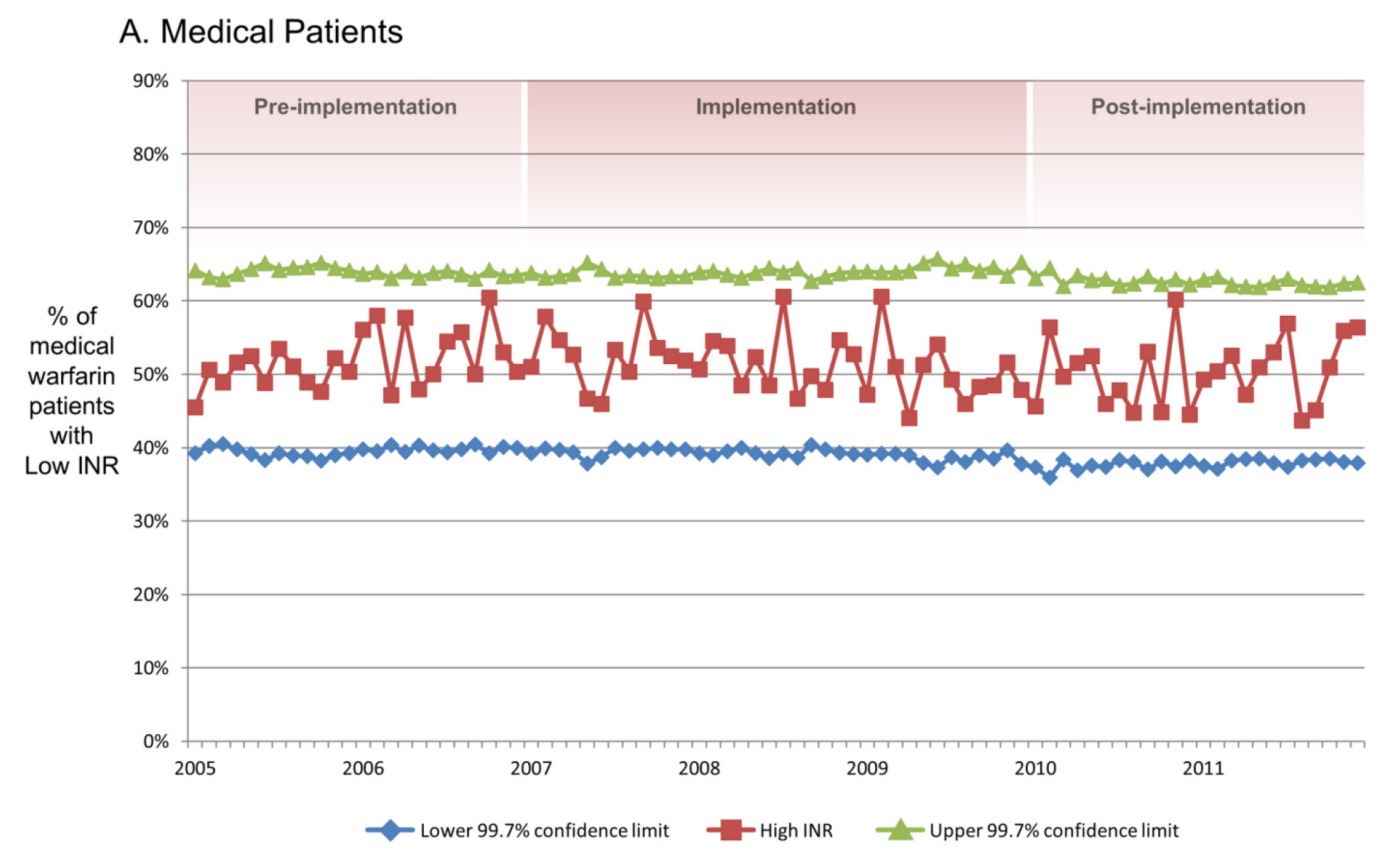

\section{B. Surgical Patients}

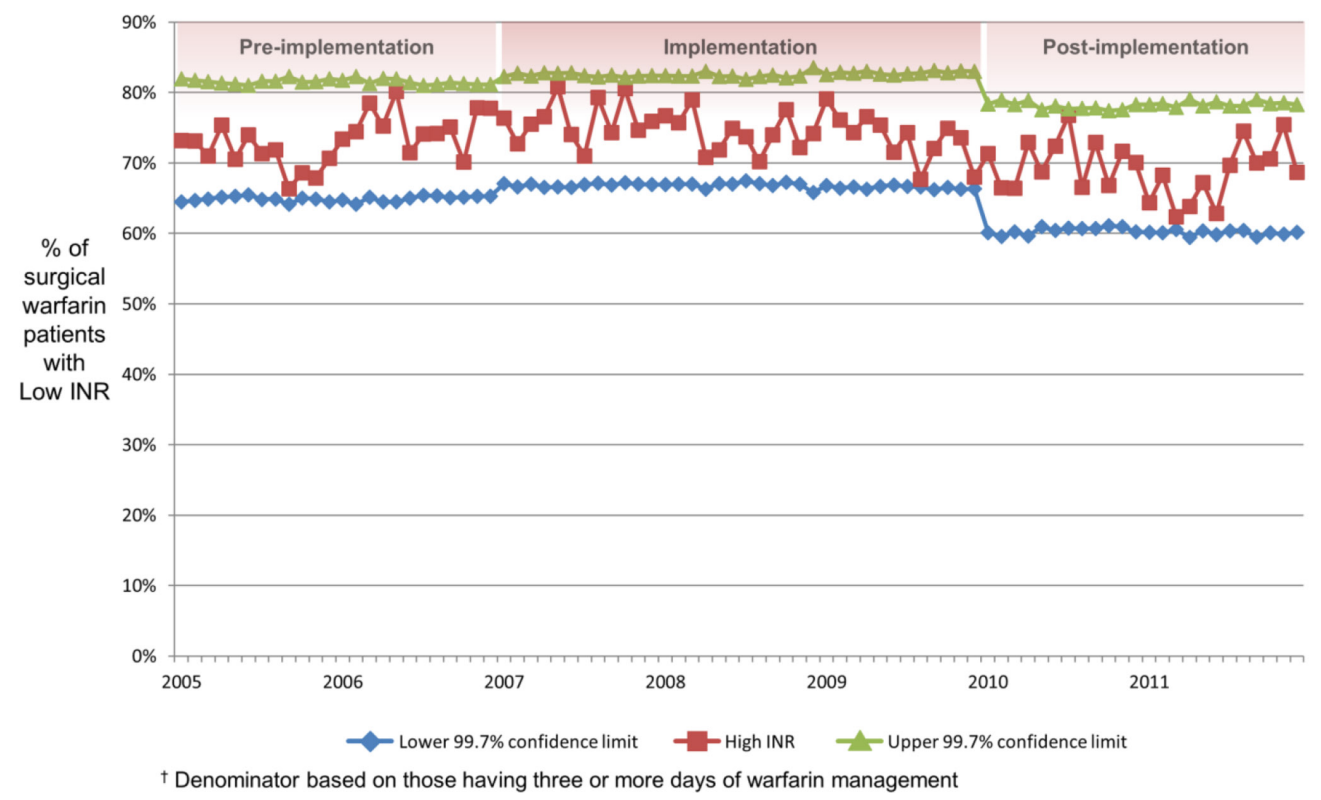

Figure 3 Per cent of inpatient warfarin recipients with low international normalised ratio $($ INR) $(<1.7)$ at the third day $\uparrow$ from preimplementation to postimplementation phases.

with the use of a computer-generated dosing process (108 patients) and found a significant reduction in the proportion of INR $\geq 4$ from $7.0 \%$ to $2.5 \%$, respectively $(\mathrm{p}=0.004){ }^{22}$ Using a prospective, cluster randomised design, Schillig et al evaluated the impact of a pharmacist-directed anticoagulation service (PDAS) (250 patients) compared with usual care (250 patients) on a composite endpoint of any INR $>5$, major bleeding, or new thrombosis assessed during the hospitalisation to 30 days after discharge. ${ }^{23}$ Although not reaching significance, the results suggest that the direction of effect favoured the PDAS and that reduction in INR $>5$ was the driver of this effect. Finally, Dawson et al implemented an inpatient pharmacist warfarin management programme and demonstrated a decrease in the rates of INR $>5$ from $7.85 \%$ to $1.85 \%$ ( $\mathrm{p}=0.004$ ) among 293 baseline patients under usual care and 217 intervention patients under pharmacist management, respectively. ${ }^{24}$ The importance of vigilant warfarin monitoring in the hospital is also demonstrated by Metersky et al, who showed that any 1 or 2-day omission of INR measurement was a hazard for over-anticoagulation. ${ }^{25}$ By our protocol, INR measurement was ordered daily and only omitted or reduced in frequency in rare situations. 
Table 2 Comparison of outcome measures before and after implementing a pharmacist-managed warfarin order set among hospital patients receiving warfarin

\begin{tabular}{|c|c|c|c|}
\hline \multicolumn{4}{|c|}{ A. Medical patients } \\
\hline \multirow{2}{*}{$\begin{array}{l}\text { Outcome } \\
\text { measure }\end{array}$} & $\begin{array}{l}\text { Preimplementation } \\
(\mathrm{n}=7200)\end{array}$ & $\begin{array}{l}\text { Postimplementation } \\
(\mathrm{n}=6856)\end{array}$ & \multirow[b]{2}{*}{$P$ values } \\
\hline & n (\%) & n (\%) & \\
\hline High INR & $399(5.6)$ & $237(3.4)$ & $<0.001$ \\
\hline Low INR* & $1888(51.8)$ & $1772(50.4)$ & 0.332 \\
\hline Bleeding & $109(1.5)$ & $125(1.8)$ & 0.152 \\
\hline DVT & $5(0.1)$ & $10(0.2)$ & 0.201 \\
\hline PE & $7(<0.1)$ & $3(0.1)$ & 0.345 \\
\hline Stroke & $5(0.1)$ & $15(0.2)$ & 0.024 \\
\hline MI & $18(0.3)$ & $8(0.1)$ & 0.078 \\
\hline $\begin{array}{l}\text { Any } \\
\text { thrombosis }\end{array}$ & $35(0.5)$ & $35(0.5)$ & 0.905 \\
\hline Hold warfarin & $1160(16.2)$ & $1090(15.9)$ & 0.959 \\
\hline $\begin{array}{l}\text { Missing INR } \\
\text { on any day }\end{array}$ & $4703(65.3)$ & 2059 (30.0) & $<0.001$ \\
\hline \multicolumn{4}{|c|}{ B. Surgical patients } \\
\hline \multirow{2}{*}{$\begin{array}{l}\text { Outcome } \\
\text { measure }\end{array}$} & $\begin{array}{l}\text { Preimplementation } \\
(\mathrm{n}=8673)\end{array}$ & $\begin{array}{l}\text { Postimplementation } \\
(\mathrm{n}=9109)\end{array}$ & \multirow[b]{2}{*}{$P$ values } \\
\hline & n (\%) & n (\%) & \\
\hline High INR & $451(5.2)$ & $219(2.4)$ & $<0.001$ \\
\hline Low INR* & 6349 (73.2) & 3972 (69.3) & $<0.001$ \\
\hline Bleeding & $1170(13.5)$ & $1011(11.1)$ & $<0.001$ \\
\hline DVT & $20(0.2)$ & $8(0.1)$ & 0.016 \\
\hline PE & $16(0.2)$ & $8(0.1)$ & 0.079 \\
\hline Stroke & $23(0.3)$ & $26(0.3)$ & 0.797 \\
\hline MI & $49(0.6)$ & $42(0.5)$ & 0.332 \\
\hline $\begin{array}{l}\text { Any } \\
\text { thrombosis }\end{array}$ & $105(1.2)$ & $80(0.9)$ & 0.029 \\
\hline Hold warfarin & 1925 (22.2) & $1148(12.6)$ & $<0.001$ \\
\hline $\begin{array}{l}\text { Missing INR } \\
\text { on any day }\end{array}$ & $6071(70.0)$ & $3680(40.4)$ & $<0.001$ \\
\hline
\end{tabular}

${ }^{*}$ Denominator based on those having 3 or more days of warfarin management.

DVT, deep vein thrombosis; INR, international normalised ratio; MI, myocardial infarction; PE, pulmonary embolism.

The impact on bleeding complications was perhaps less than expected at the outset of the project. While a supratherapeutic INR increases the risk of bleeding, other risk factors for warfarin-related haemorrhage were not assessed by multivariate analysis. The primary aim of our project remains valid, as an excessively supratherapeutic INR is a modifiable risk factor. A potential reason the medical bleeding rate did not drop is that this group includes short-stay cardiology patients. These patients may have only received one or two warfarin doses before dismissal. We also did not assess bleeding events postdischarge through readmissions or emergency visits for any patients. We would not expect these factors to have been distributed unequally in the preimplementation and postimplementation periods but this was not directly evaluated. Despite a decrease in bleeding rates for surgical patients, adoption of the protocol was less for surgical than for medical patients. This may have been due to the presence of short-stay surgical patients or due to surgeons not wanting to delegate anticoagulation care. Investigations to understand barriers to adoption by medical and surgical providers could be the subject of future improvement efforts. Finally, the criteria for bleeding events may have contributed to our findings. In contrast to bleeding definitions from the International Society on Thrombosis and Hemostasis (ISTH) we set a greater haemoglobin drop criterion $(4 \mathrm{~g} / \mathrm{dL})$ when compared with the ISTH $(2 \mathrm{~g} / \mathrm{dL}){ }^{26} 27$ We also identified patients receiving blood products for warfarin reversal in our criteria; this may have been inappropriate in some cases if given to facilitate urgent/ emergent procedures. Again, there is no a priori reason to expect a differential effect between preimplementation and postimplementation.

Implementation of the PMWP created a possible educational trade-off. By ceding warfarin dosing to the pharmacists, trainees could possibly be less adept at warfarin management in their future practices. Project leadership considered this to be an acceptable opportunity cost as healthcare is increasingly delivered by teams-in which physicians rely on others' expertise to enhance patient safety. Important institutional lessons learnt include the recognition that the time to implement the PMWP was long. As a result, institutional leadership endorsed future approval to spread improvements through a centralised but representative group to accelerate spread. The importance of project management in the drive to success through DMAIC was also recognised.

\section{Limitations}

Our analysis has several important limitations. The ability of providers to opt in or not potentially introduces selection bias. In this large-scale retrospective analysis, we did not compare the frequencies of excessive supratherapeutic INR between those patients managed through the PMWP and those who were not. Providers whose patients were not managed under the PMWP were not blinded to pharmacist management or to the institutional emphasis on warfarin safety; and pharmacists perhaps felt empowered to influence the care of non-protocol patients. However, our segmented regression analysis at the institutional level assesses the impact of the implementation and would minimise this issue. We also did not control for factors affecting INR control between the preimplementation and postimplementation periods-for example, a change in the presence of interacting medications. The extent to which our process improvement project could be adopted by other hospitals may be limited by their available resources to create a similar inpatient process for warfarin management. We had information technology systems and personnel to embed the warfarin management algorithm into the workflow of busy inpatient unit pharmacists and had adequate staffing to ensure that the workload could be accommodated. 


\section{CONCLUSIONS}

The design, implementation and results of our inpatient warfarin management improvement project contain useful lessons for other practices and institutions. Even if information technology resources are limited at a particular facility, standard tools for dosing and consolidation of care under pharmacy should be feasible. If process changes are piloted and implemented gradually, with attention to pharmacist workload, similar processes should be achievable and sustainable. Finally, the centralisation of inpatient management within specialty pharmacy-led practice could enable improvements in safety and quality metrics related to all anticoagulants, not just warfarin- expanding the impact of the innovation across a group of medicinal agents among the highest risk drugs given to our patients.

Acknowledgements The authors appreciate the assistance of Ruth M Boland, RN, and Peter W Svendsen. The authors also wish to recognise the efforts of Timothy $\mathrm{J}$ Brennan, MBA, and Christa Y Leung, MBA.

Contributors PRD planned the study design, participated in the analysis of results and drafted the manuscript. JPM and JMN planned the study design, participated in the analysis of results and reviewed the manuscript. DM, JBC, NNO, JGOM and DLR participated in the analysis of results and reviewed the manuscript.

Funding The quality improvement efforts were funded through routine clinical practice activities. Retrospective analysis was partially supported through the Mayo Clinic Robert D and Patricia E Kern Center for the Science of Health Care Delivery.

Competing interests None declared.

Patient consent Obtained.

Provenance and peer review Not commissioned; externally peer reviewed.

Open Access This is an Open Access article distributed in accordance with the Creative Commons Attribution Non Commercial (CC BY-NC 4.0) license, which permits others to distribute, remix, adapt, build upon this work non-commercially, and license their derivative works on different terms, provided the original work is properly cited and the use is non-commercial. See: http://creativecommons.org/ licenses/by-nc/4.0/

(C) Published by the BMJ Publishing Group Limited. For permission to use (where not already granted under a licence) please go to http://www.bmj.com/company/ products-services/rights-and-licensing/

\section{REFERENCES}

1. Ageno W, Gallus AS, Wittkowsky A, et al. Oral anticoagulant therapy: antithrombotic therapy and prevention of thrombosis, 9th ed: American college of chest physicians evidence-based clinical practice guidelines. Chest 2012;141:e44S-e88S.

2. Kirley K, Qato DM, Kornfield R, et al. National trends in oral anticoagulant use in the United States, 2007 to 2011. Circ Cardiovasc Qual Outcomes 2012;5:615-21.

3. U.S.Department of Health and Human Services, Office of Disease Prevention and Health Promotion. Section 5: anticoagulants. National action plan for adverse drug event prevention. Washington, DC: U.S.Department of Health and Human Services, Office of Disease Prevention and Health Promotion, 2014.

4. Barnes GD, Lucas E, Alexander GC, et al. National trends in ambulatory oral anticoagulant use. Am J Med 2015;128:1300-5.

5. Huddleston JM, Diedrich DA, Kinsey GC, et al. Learning from every death. J Patient Saf 2014;10:6-12.
6. Hartis CE, Gum MO, Lederer JW. Use of specific indicators to detect warfarin-related adverse events. Am J Health Syst Pharm 2005;62:1683-8.

7. Rozich JD, Haraden CR, Resar RK. Adverse drug event trigger tool: a practical methodology for measuring medication related harm. Qual Saf Health Care 2003;12:194-200.

8. Lucado J, Paez K, Elixhauser A. Medication-Related Adverse Outcomes in U.S. Hospitals and Emergency Departments, 2008: Statistical Brief \#109. Rockville, MD: Healthcare Cost and Utilization Project (HCUP) Statistical Briefs, 2006.

9. Federico F. Preventing harm from high-alert medications. Jt Comm J Qual Patient Saf 2007;33:537-42.

10. Dager WE, Branch JM, King JH, et al. Optimization of inpatient warfarin therapy: impact of daily consultation by a pharmacistmanaged anticoagulation service. Ann Pharmacother 2000;34:567-72.

11. Bond CA, Raehl CL. Pharmacist-provided anticoagulation management in United States hospitals: death rates, length of stay, Medicare charges, bleeding complications, and transfusions. Pharmacotherapy 2004;24:953-63.

12. Goodman D, Ogrinc G, Davies L, et al. Explanation and elaboration of the SQUIRE (Standards for Quality Improvement Reporting Excellence) Guidelines, V.2.0: examples of SQUIRE elements in the healthcare improvement literature. BMJ Qual Saf 2016;25:e7.

13. Berg TM, O'Meara JG, Ou NN, et al. Risk factors for excessive anticoagulation among hospitalized adults receiving warfarin therapy using a pharmacist-managed dosing protocol. Pharmacotherapy 2013;33:1165-74.

14. Agency for Healthcare Quality and Research. AHRQ Q/ version 4.5, patient safety indicators \#4, technical specifications, death rate among surgical inpatients with serious treatable complications, 2013.

15. Mehran R, Rao SV, Bhatt DL, et al. Standardized bleeding definitions for cardiovascular clinical trials: a consensus report from the Bleeding Academic Research Consortium. Circulation 2011;123:2736-47.

16. Agency for Healthcare Quality and Research. AHRQ QI version 4.5, patient safety indicators \#9, technical specifications, perioperative hemorrhage or hematoma rate, 2013.

17. Moriarty JP, Daniels PR, Manning DM, et al. Going beyond administrative data: retrospective evaluation of an algorithm using the electronic health record to help identify bleeding events among hospitalized medical patients on Warfarin. Am J Med Qual 2017;32.

18. Lawthers AG, McCarthy EP, Davis RB, et al. Identification of in-hospital complications from claims data. Is it valid? Med Care 2000;38:785-95.

19. Naessens JM, Huschka TR. Distinguishing hospital complications of care from pre-existing conditions. Int J Qual Health Care 2004;16(Suppl 1):i27-i35.

20. Naessens JM, Scott CG, Huschka TR, et al. Do complication screening programs detect complications present at admission? $\mathrm{Jt}$ Comm J Qual Saf 2004;30:133-42.

21. Wong YM, Quek YN, Tay JC, et al. Efficacy and safety of a pharmacist-managed inpatient anticoagulation service for warfarin initiation and titration. J Clin Pharm Ther 2011;36:585-91.

22. Gouin-Thibault I, Levy C, Pautas E, et al. Improving anticoagulation control in hospitalized elderly patients on warfarin. J Am Geriatr Soc 2010;58:242-7.

23. Schillig J, Kaatz S, Hudson M, et al. Clinical and safety impact of an inpatient pharmacist-directed anticoagulation service. $J$ Hosp Med 2011;6:322-8.

24. Dawson NL, Porter IE, Klipa D, et al. Inpatient warfarin management: pharmacist management using a detailed dosing protocol. $J$ Thromb Thrombolysis 2012;33:178-84.

25. Metersky ML, Eldridge N, Wang Y, et al. Predictors of warfarinassociated adverse events in hospitalized patients: Opportunities to prevent patient harm. J Hosp Med 2016;11:276-82.

26. Schulman S, Kearon C. Subcommittee on Control of Anticoagulation of the Scientific and Standardization Committee of the International Society on Thrombosis and Haemostasis. Definition of major bleeding in clinical investigations of antihemostatic medicinal products in non-surgical patients. J Thromb Haemost 2005;3:692-4.

27. Schulman $S$, Angerås $U$, Bergqvist $D$, et al. Definition of major bleeding in clinical investigations of antihemostatic medicinal products in surgical patients. J Thromb Haemost 2010;8:202-4. 